\title{
A qualitative study of the emotional coping and support needs of children living with a parent with a brain injury
}

Poul Rohleder, School of Psychology, University of East London, P.A.Rohleder@uel.ac.uk John Lambie, Department of Psychology, Anglia Ruskin University, john.lambie@anglia.ac.uk Emily Hale, Department of Psychology, Anglia Ruskin University, United Kingdom

Accepted 15 August for publication in Brain Injury

This article may not exactly replicate the final version published in the journal. 


\section{Abstract}

Primary objective: To examine emotional coping and support needs in children of persons with acquired brain injury, with a view to understanding what interventions would be helpful for these children.

Design: The study was qualitative, using a thematic analysis approach.

Methods and procedure: Six children between 9 and 18 years of age, six parents (three with $\mathrm{ABI}$ ), and three support workers were interviewed either at home or at a support centre, using a semi-structured interview guide.

Results: Children reported using a variety of adaptive and maladaptive emotional coping strategies, but were consistent in expressing a need for credible validation, i.e. sharing experiences with peers. The results are presented under four overarching themes: difficulties faced; emotions experienced; coping strategies; and reported support needs.

Conclusions: The results reveal an interaction between the child's experiences of complex loss that is difficult to acknowledge, emotional distancing between parent and child, and the children's need for credible validation. All children expressed a desire for talking to peers in a similar situation to themselves, but had not had this opportunity. Interventions should set up such peer interaction to create credible validation for the specific distress suffered by this population.

Key words: children, parents, ABI, emotion, emotion regulation, coping, validation. 


\section{Introduction}

There is a small but growing literature on the psychological effects of parental brain injury on children [1-5]. It is known that having a parent with acquired brain injury is a stressor that can be associated with increased symptoms of depression [5], posttraumatic stress [3], and behavioural problems [4] in children, and that these problems are partly mediated by poorer relationships with both the injured and the non-injured parent [4-5].

Parental brain injury may be particularly traumatic for children since it involves interpersonal loss - typically including chronic disabilities in a parent and changes in parent-child relationships [6]. Thus there is a double loss, since one parent now has a brain injury and the other parent is pre-occupied with their care [1]. Coping with the trauma of a parent's brain injury is thus made especially hard because the child's main support network - their parents - has also become diminished by the trauma.

Partly because of this complexity it is still not clear from the literature what kinds of support children in this population need and what would be the most effective interventions for them. The present research adopts an exploratory qualitative design to investigate the range of different coping styles used by a small sample of children in this population and what kinds of support they feel would help them.

\section{Coping with Parental Brain Injury}

Coping refers to the actions and thoughts we use to deal with stressful situations or difficult emotions [7]. What are the particular stresses and difficult emotions faced by children in this population? This question is complicated because it is clear from the 
literature that many children in this group find it very difficult to talk about their negative emotions and that there is some denial, ambiguity, and "hidden" loss [1-2,8]. There is evidence that there are increases in sadness, anger, and worrying [2], and post-traumatic stress symptoms [3], but also that there are difficulties in coping with contradictory emotions, for example, confusing mixtures of love, anger and sadness [8]. Other themes that have emerged from qualitative interviews include fear of losing the injured parent [2], a sense of social isolation [8], fears of family disintegration, and coping with increased aggression from the injured parent [1].

Thus, there is a complicated set of difficult feelings and family dynamics that the child has to cope with. However, little is known about the coping strategies used by children in this population and whether or not they are successful. KiefferKristenson \& Johansen [2] reported five coping strategies used by a sample of fourteen 7-14 year olds with a parent with a brain injury: distraction, helping others, independence, backing out of overwhelming situations, and being positive, but it is unclear how effective these strategies were. The most commonly used was distraction (e.g. socializing, leisure activities, listening to music, etc.), and this was described as "an effective tool", but it should be noted that research on the effectiveness of distraction in coping with negative emotions in general is mixed. For example, Mendolia \& Kleck [9] found in a normal adult population that those who talked about their feelings after a stressful event had more arousal in the short term than those who distracted themselves, but these effects were reversed after 48 hours with those who used distraction showing more arousal when re-exposed to the stressor than those who used talking.

Generally, research suggests that some styles of coping are more effective than others. In a large meta-analysis [10] of the relationship between different emotion 
regulation strategies and psychopathology (using data from both adult and child populations), it was found that the more protective strategies were reappraisal, problem-solving, and acceptance and the less effective strategies were suppression, rumination, and avoidance. In addition to these, talking or writing about one's emotions has been found to be effective [9], and so has distraction [11], though see the caveat above. Not much is known about the extent to which children of parents with brain injuries use the range of these different coping strategies, so one of the aims of the present study is to specifically explore this.

\section{The Child's Support Needs}

What are the support needs of these children and what is known to help them? Although social support is known to be important for families after head injury [12], previous research has found that children in this population are reluctant to reach out for support beyond their immediate family [1]. For example, Kieffer-Kristensen \& Johansen [2] found that the primary support used by children was the parent not affected by ABI, followed by siblings and grandparents. Although many had been offered support from teachers, most said they did not take this up as they didn't want to be treated differently and didn't think that teachers really understood their situation.

There is very little research on what interventions are successful for children living with a parent with ABI. Evans-Roberts et al [13] recently reviewed the research on psychological interventions for families following acquired brain injury, and almost all the research measured outcomes in caregivers, spouses, or the patients themselves. Only one intervention study [8] reported detailed outcomes on the children of brain injured persons. They carried out a pilot multifamily group intervention study with six families affected by brain injury. Participants took part in 
family therapy as a whole group and in additional parent-only or child-only subgroups. The six families met for 12 sessions over 6 months, with quantitative and qualitative measures taken pre and post group, and at 3 months follow up. 11 parents and 9 children took part in the study with the children's ages ranging from 4-14 years. Children's emotional and behavioural distress as measured by the Behaviour Assessment Systems for Children did not show a strong decrease after family therapy, although there was some evidence that children were under-reporting or denying distress and that this may have been more prominent at the outset of the study. Overall, families reported that the multifamily group sessions were helpful, and the children reported that they liked meeting other children who shared similar problems. However, Charles et al [8] do not report in detail what specifically the children felt they needed to help them cope.

\section{Rationale for the Present Study}

It is clear that children of parents with brain injury suffer a complex and ambiguous kind of loss, but how to best help them cope with this loss is less clear. Recent research has concluded that there is a "need for professionals to talk to the children of injured parents about their experiences of loss and grief' [2, p. 8], but the children's reluctance to talk about negative emotions, and their reluctance to talk to people outside the family may make this kind of solution problematic. There is a need to explore in more detail what support the children feel they need.

Furthermore, the support needs of the children partly follow from how they are coping, or trying to cope, and whether they are coping effectively. But there has been little research addressing the range of coping strategies that children in the population are using, and if these strategies are effective. 
The present study is a pilot investigation designed to explore and raise awareness of three key questions: what are the difficulties faced by children with a parent with brain injury, what are the different emotion coping strategies they use, and what are the support needs of such children? A further aim is to draw inferences from the answers to these questions about suitable interventions for these children.

\section{Method}

The study utilized a qualitative research design and collected data from three groups of participants: Children of parents with ABI, the children's parents and community support workers. Collecting data from these three perspectives allowed for triangulation of data and improved validity [14]. Ethical approval was provided by the authors' institutional research ethics panel. All aspects of the study were also discussed with senior staff at the charity organisation through which participants were recruited.

\section{Participants}

Participants were recruited through a charity organisation in the East of England that support individuals who have suffered a brain injury. Families were sought, where a parent had suffered a brain injury, and where they had children aged 7 to 16 . Children under the age of 7 were excluded as they may be more likely to withhold information about negative emotions [15] and will have more difficulties with articulating emotion regulation strategies. The upper limit of 16 years was chosen as a conventional boundary for childhood. This was extended for two children who were over the age of 16 at the time of the interview (ages 17 and 18), but had been 16 or younger at the time the parent had acquired a brain injury. 
Staff at the charity approached families that they support informing them of the study and whether they would be interested in being involved. If so, contact details were passed on to the researchers, who then liaised directly with the families. Four families participated in the study. A fifth family who expressed an interest were not included as the children were much younger than 7 . The parents and children in each of the four families were provided with detailed information about the study, and the consent of all parents and children were sought. Only individuals with ABI who had capacity to consent were recruited.

In order to ensure confidentiality and anonymity, we will provide a general description of the sample. A total of six children/teenagers (two males and four females; aged between 9 and 18) were interviewed from 4 families. At the time of parental ABI, the ages of the children were between 4 and 16 . One child was born to a parent (mother) who had acquired brain injury. Of the 4 families, 2 were where the father had the brain injury, and 2 where the mother had the brain injury. For two families both parents took part in the interview. In the other two families, only the mother (one who had an ABI) took part in the interview. Additionally, three community support workers from the charity were recruited as key informants. These were support workers who had some experience of supporting individuals with ABI who had young children.

\section{Interviews}

Interviews took place in the family's home, or in confidential rooms at the charity organisation. After an initial meeting with the family to discuss the aims of the project and get signed consent, parents and children were interviewed simultaneously in different rooms. Interviews with parents were conducted by PR. Where both parents 
participated, they were interviewed together. Interviews were semi-structured and lasted approximately 45 minutes to 1 hour. Questions focused primarily on the parents impressions and understanding of how their children had responded to and emotionally coped with the parental brain injury, the parents' experience of parenting, and what support resources had helped them or were lacking.

Interviews with the children were conducted by JL. Where there were siblings, each child was interviewed separately. Interviews were semi-structured and lasted approximately 30 minutes to 45 minutes. Questions focused on their recalled experience of having to cope with their parent's brain injury, what emotions they have experienced, what emotional coping strategies they have used, and what support resources had helped them or were lacking. For younger children, an emotions chart and a coping with emotions chart was used as a prompt. These charts were modified versions of ones available from an on-line cognitive-behavioural therapy resource (www.getselfhelp.co.uk), and were adapted to be more suitable for children.

Interviews with support workers were conducted by EH. Interviews were semi-structured and lasted approximately 45 minutes to 1 hour. Questions focused on their impressions (drawing from their experience) of how children cope where a parent has suffered a brain injury, what do parents and/or children disclose to them about the child's coping, what challenges they have faced in supporting families.

All interviews were audio-recorded, with consent, and transcribed verbatim. Families were informed that we would not share with them what the children or the parents had shared in their respective interviews, in order to protect confidentiality. 


\section{Analysis}

All interviews were analysed by means of thematic analysis [16]. Transcripts were analysed using a combination of a deductive and inductive approach, identifying themes related to the focus of the research questions as well as what emerged from the data. In order to enhance validity, one transcript from a parent, a child and a support worker were independently analysed by PR and JL, and emerging codes and themes discussed and compared. With some core emerging codes and themes identified, all other transcripts were analysed, and codes and themes added as they emerged in the data. An additional content analysis was conducted on the interviews transcripts of children, in order to quantify how many of the children had experienced particular emotions or utilized particular coping strategies. The analysis and emerging results were frequently discussed and agreed upon as a team.

\section{Results}

We present the results under the four core themes of: difficulties faced; emotions experienced; coping strategies; and reported support needs. Sub-themes are presented within each of these. Following the process of triangulation, we will point out convergences in the analysis of data from the three groups of participants.

\section{Difficulties Faced}

In terms of difficulties faced by the children with living with a parent with a brain injury, four broad themes were identified: increases in parental anger; loss of care and attention from parents; difficulties with communication; and people not truly understanding. 
Increases in parental anger. Most of the children (5 out of 6) spoke of difficulties with increased anger in their parent post brain-injury. The children spoke about this as a noticeable difference, which they found particularly difficult to cope with. For example, one daughter described how her father "loses his temper a bit more" and how she sometimes found that "quite hard". Likewise a son described his father's change in expression of anger:

'He got more irate, more things wound him, well the same things wound him up but he just made a bigger deal out of it, like you know not doing household chores or not going to bed when you're told too and he'll flip out'. (Family $2-$ son)

In some cases this was reported to lead to more parental conflict:

'he never seemed in a good mood. And, a lot, lots of times they were arguing and it's such a small house it's quite hard not to hear, and that was quite upsetting, yeah I think that was probably one of the worst bits.' (Family 2 daughter)

This was corroborated by the reports from support workers who spoke about how they have observed the difficulty children have dealing with issues of anger. This included dealing with the changes in personality in the injured parent:

'certainly one particular father that I spoke to, his daughter, I think she was about 2 years old and found it really difficult to comprehend kind of why daddy lost his temper so quickly'. (Support Worker 1)

But support workers and parents also spoke about the observed and reported anger of the child (see below).

Loss of care and attention from parents. This was a theme mentioned by half our sample, and it was related to losses of both practical and emotional support. While 
there was some mention made in a minority of interviews about some positive changes (for example two children referred to how their injured father had become gentler emotionally), this was overridden by an overall sense of loss. This loss was spoken about in different ways and in relation to both the parent with the brain injury, and the non-injured parent. With regards the parent with the brain injury, the children spoke about having experienced a loss of attention and care from the injured parent. One daughter spoke about incidents where she felt a little neglected as a result of her mother's cognitive difficulties:

'I feel that she can look after me most of the time, but like the other day - I know that I'm 12 and I can get my own dinner and do that, I'm not the best, ..., cos now she's sort of forgot I needed to eat. She forgot I needed dinner.' (Family 3 - daughter)

One of the sons spoke about a sense of loss of having lost a "father figure", even though his father was present:

'that's kind of difficult cos I guess I see him as a friend and rather than a dad cos of what he's gone through.' (Family 2 - son)

But some of the children also spoke about sensing a loss of attention and care from the non-injured parent who was having to cope with their partner's brain injury. For example, one daughter stated:

'I guess at the time I felt like I had just lost one parent I have kind of ...[also] lost my mum, but she was kind of not there because she was with him at the hospital for just like maybe three months or so while it was really serious'. (Family 1 - daughter) This loss of parental attention was a theme that also came up in the interview with parents and with the support workers. The support workers commented on this in 
relation to the 'loss' of the parent with a brain injury. The parents recognised that their children may have struggled with the loss of attention from both parents. All three of the parents interviewed who had not suffered a brain injury, commented on their feelings of guilt and concern that they were very occupied with having to deal with the crisis of their partner's brain injury, and in retrospect felt that they, out of necessity, were more absent for their children that usual. For example, one mother stated how she had to leave her teenage children to get on with things on their own: 'because we were going to the hospital all the time there wasn't really any time to, they kind of just got on with things, so I don't know how much time there was for me to be there with them to look at what's happening'. (Family 2 - mother)

Similarly a father (Family 4) thinks back on how absent he might have been during the first few months and said how he "wasn't the dad I used to be, I was totally preoccupied". One mother spoke about how this had an effect on their child, who felt a loss of two parents (her father with a brain injury and her mother):

'I spent a lot of time at the hospital and one of the things that my daughter has since said, which at the time I didn't even realise at all was that she said "you stopped reading to me at bedtime" [...] I realised that that really had and still has had an impact on her so that time away of course they were not only concerned about [father] but also I wasn't around very much and it was at a time when they probably needed me around more'. (Family 1 - mother) As a result of the trauma of the brain injury and the relative absence of the parent(s), some parents reflected on how in many ways their child(ren) had "to grow up quicker" and take on responsibilities over that expected of a child their age. This was reflected in the interview with one daughter who spoke about having to care for 
herself and make herself dinner (see above). This was also observed by the support workers. For example, one stated:

'the children take on a bit of a caring role inadvertently, you know, kind of just automatically do it. You know it might be that they have to do more washing up or, you know, or get them sandwiches for school or whatever it may be'. (Social Worker 1)

Difficulties with communication. Some participants mentioned problems with speech or pragmatics in the parent with a brain injury as being difficult to deal with. One son described communication with his father as being "like a constant game of charades" (Family 1 - son) due to his father's difficulties in pronouncing words. One daughter noticed this as a change in her mother's personhood:

‘family jokes - she didn’t find them funny anymore. Or simple things like sarcasm -- doesn't understand it, or just not being herself really... to see someone in your mum's body but she's not the person she was before. And you can't, you can't understand why it's not the same person' (Family 4 daughter)

This was related to the theme of loss in the sense that children spoke about the brain injured parents as 'being there, but not there' in the way they had known them previously.

People not truly understanding. A theme commonly reported by some of the older children was finding it difficult when other people showed a lack of understanding. For example, one daughter spoke about her frustration at other people's comments about the recovery of the brain injured parent, which to her did not consider the ongoing challenges: 
'I think the hardest thing is not having people around you who understand what it's like. Because, ... you'd have a lot of family members saying it's brilliant she's recovered, she's done so well. But from living at home with her and being with her all the time, you know that's not the case... I think that's so hard, I think, that's probably the hardest thing for me is explaining to people that she's not the same person, and she's not recovered fully'. (Family 4 - daughter)

Other children spoke about the assumptions that others may hold about the impact that the brain injury has on the injured parent, or on the child themselves. For example, one son spoke about how any difficulty he may have had with school work was related to this:

'people kept assuming that if I didn't the right test results or I didn't get the right grades or I wasn't performing at the levels that I was expected too, it was because of... grief over you know the injury, people kept assuming that was the case, but it wasn't.' (Family 2 - son)

Another daughter spoke about the assumptions others had about the ability of the brain injured parent:

‘... people like my granny... she always says really unhelpful stuff. Because mum travels quite a bit, she will say you got to make sure you look after family ...[But] just because he has had a brain haemorrhage doesn't mean he is incapable of doing stuff.' (Family 1 - daughter) 


\section{Emotions}

Children were specifically asked about five emotions in relation to their feelings about living with a parent with $\mathrm{ABI}$ : anger, sadness, love, guilt, and anxiety. Figure 1 shows a count of how many participants agreed they had felt that emotion.

[insert Figure 1 here]

\section{Worry and anger}

The support workers and parents commented on the observed emotional reactions of the children. The support workers primarily spoke about the children's anger. Parents gave a more nuanced account of the emotions they observed in their children. Parents reported that their children's initial reactions to their parent having a brain injury, was fear and confusion. Subsequent reactions reported were anger, anxiety/worry, loss/grief and embarrassment. Many of the parents observed these reactions in terms of the child's withdrawal, noticing them being preoccupied with something. They usually understood this as the child being worried/anxious or sad. For example, one parent (with an acquired brain injury) reported being aware that her daughter might be struggling with some emotions because the daughter would become quiet:

'Sometimes she gets upset and just says to me I'm scared, but normally she'll go quiet and look, and she's just not her normal bubbly self, and I can see in her eyes that there's something wrong'. (Family 3 - mother) The parents seemed to more readily report (and perhaps recognise) their child's expressions of fear, worry and also anger. For parents of teenagers, they struggled to 
differentiate what was typical teenager emotional expressions (e.g. their child being oppositional) and what was related to the difficulties around the brain injury.

It is notable that, while all children reported feeling angry and could give examples of anger they had felt, only half the children reported feeling sad about their situation.

Struggles with loss. While only three children responded to feeling any sadness when specifically prompted, their interview narratives indicated a possible struggle with a sense of loss and sadness. When asked, two of these children initially reported having felt no sadness, but sadness then emerged later in the interview. For example, when one of the daughters interviewed was initially asked "were there times you felt sad?", she replied "No", and also stated"positives massively outweigh the negatives which is obviously not how it works for so many people". However, later towards the end of the interview she gave this anecdote:

'It's really little things but mum was at the hospital every night and from because I said I was nine, I think I have had a story read to me every night since I was young and that stopped and that's something that we have talked about since but it's still really upsets me that I didn't get a story.' (Family 1 daughter)

Similarly, one son who was interviewed made several statements about not being negatively affected and therefore not needing to engage in coping: "It never really affected me on a kind of really deep level...”; “...there was never a deep revelation where I realised that actually this is the issue... it never really affected me, work or personal life never brought it up"; "I think there was never anything for me to cope with”. But, immediately after that last statement, he seemed to contradict himself: 
'I guess it's difficult; the biggest issue was lack of father figure I guess, because it was at that stage that's kind of difficult cos I guess I see him as a friend rather than a dad. So that was difficult to cope with, I don't know how I coped with it. Maybe I haven't, I don't know, but that was the most difficult aspect to cope with.' (Family $2-$ son)

Emotions of sadness were also indirectly referred to in the children's accounts of having felt a sense of loss in their parent's attention, care and communication (see themes above).

Parents also seemed to suggest that it was the feelings of sadness and loss that were more difficult to acknowledge and talk about. As one mother stated:

'I think the biggest one that is still there, I wonder if it's just sadness. You know, when we all get together and we have to leave him or the unusualness of it. I think it's the sadness.' (Family 2 mother)

\section{Emotional coping strategies}

The children were specifically asked about whether they used any of 6 coping strategies: behavioural avoidance, suppression of emotion, distraction, talking to others, relaxation/meditation, and reappraisal. These were phrased in an ageappropriate terminology and were illustrated in pictures. The number of children agreeing that they used each strategy is shown in Table 1. In all cases, they were asked and were able to give a specific example of when they had used the strategy.

[insert Table 1 here] 
Children used a range of "helpful" and "unhelpful" coping strategies (as specified by the meta-analysis of Aldao et al [10]). Two unhelpful strategies - avoidance and suppression - were used by most children. One helpful strategy - talking to others was also used by most children, although only 1 child reported talking about their difficulties to someone outside of their immediate family (this matches a similar finding by Charles et al [8]). Few of the children reported using relaxation techniques or reappraisal - both thought to be helpful coping strategies.

The parents and support workers did not refer to any specific coping strategies that they could observe their child using. However, the strategy of avoidance and suppression is implicit in some of the parents' observations of their child being quiet and withdrawn (see above). What the parents (and support workers) did comment on is their feeling that they needed to protect their children by shielding them from the realities of the brain injury. In a sense they did this in an attempt to help their children's coping by shielding the child from the acute stresses associated with the crisis of the injury, so as not to upset them more. However the parents struggled to decide how much of the reality they should expose their child to or share with them. For example, one mother recalled being unsure about whether she should take her young children to the hospital to see their father, who was in a coma, for fear that this may frighten them:

'I wanted someone to say "yes it's better for them to see their dad whatever the situation”, or "no don't take them in”, but I didn't feel I was getting any guidance on that at all.' (Family 1 - mother)

One of the fathers reported struggling to shield his daughter from the seriousness of her mother's head injury: 
'I tried to protect [her] as much as possible but at the same time there's no point coming home and saying everything's fine, I was trying to find that sort of right balance'. (Family 4 - father)

Some of this was the parent's concern that their child could have the space to still 'enjoy' being a child, to play and continue as best they could at school.

The support workers also commented on what they observed as the parents wanting to protect their children from some of the difficulties. One support worker reflects on how this is a balancing act, with the non-injured parent not only have to face challenges themselves, but have to support their partner and children too:

'the parent who doesn't have the brain injury is trying to support the individual with the brain injury and the children at the same time and that parent is trying to come to terms with what's happened and particularly the changes in personality and behaviour. It's really difficult for them to come to terms with it and I think sometimes we find, the conversations I've had, that try and protect the children and keep them shielded from it'. (Support worker 1)

Another support worker spoke about this in terms of the parents seeming to keep their children "in a separate box" (Support worker 3).

\section{Support needs}

In answer to questions about what kind of support the children felt would specifically help them with having to cope with their parent's brain injury, two themes emerged: sharing experiences with peers; and receiving more information or advice about living with someone with a brain injury. 
Sharing experiences with peers. This was the most commonly expressed need, with 4 of the children outlining how they would like to be able to talk to other children in a similar situation to themselves. This was also mentioned by the parents. Given the parent's own difficulties coping with the trauma of the brain injury, most parents felt that peer support for their children would be especially helpful. For example, one parent stated:

'I think just knowing that there are other people who have experienced the same thing in some ways or having some kind of way talking to someone whose not the parent, because I had my own priorities and different things'. (Family $2-$ mother) This was affirmed by one of the daughters who stated: "I think having someone to talk to who's gone through the same thing or going through the same thing" (Family 2 - daughter)

Another child (male) explained with a bit more detail:

'Yeah someone to talk, even via ... a Facebook group, or one of those kind of things where you don't necessarily need to meet the person. But I wouldn't want someone to comfort me, and put an arm around me and tell me it's all alright ... I want someone to relate to, and exchange stories with, that sort of thing.' (Family 2 - son)

As the above quote indicates, the wish was not just to have someone to talk to, but someone who could share genuine understanding and empathy through a shared experience. This was also explicitly distinguished from support through the mere receiving of information:

'I did once go to a session for children at [name of charity]. But it was just being given information. I would have preferred to talk to the other children to share stories and support each other.' (Family 4 - daughter) 
Interestingly, the two older children expressed a willingness to perhaps act as a mentor to other children in a similar situation.

All the support workers felt that they were not adequately skilled to support children's emotions or advise parents on them. One support worker said they had attempted to provide something in the past, but did not feel they had the necessary expertise and skills. As was suggested by the children and the parents interviewed, all support workers also felt that peer support would be a particularly useful, and necessary resource:

'they need that emotional support, they need that educational support they need someone to talk to they need an outlet they may need other children to meet with and talk to, I think all bases need to be covered and at different stages' (Support worker 1)

Similarly, support worker 3 stated:

'I think what we try to do a lot and what we naturally do a lot, is trying to kind of normalise stuff and to say you know this is very common, you know, to experience this. What we think would be really beneficial in this situation is to have someone to meet or talk to or see in a video, other children saying this is what I experienced, you know; my dad was like this, my dad didn't understand; perhaps give examples that they can relate to try and understand it might just make them feel more normal like they're not the only person in the world who's parent, you know, has clearly got difficulties' (Support worker 3) This support worker differentiated peer support as providing genuine understanding and empathy, as opposed to advice giving and reassurance which is all that they could provide. 
More information and advice. Wanting more information and advice due to not knowing what to expect from living with a person with brain injury was cited as a need by several of the children. For example one child stated:

'When I was younger, it would have been nice to have a list of everything that could happen, everything that will happen, and to know what's gonna happen in a few years. What's she going to be like cos I know she's not very, we don't know whether it's going to go downhill or if it's going to stay as it is, so it would be nice to know.' (Family 3 - daughter)

Similarly, another daughter stated:

'although I understood what was happening I think that it was because I was a lot older. I think for younger children, they wouldn't understand how serious it is, ... So I think for somebody to come in the house and say like this is what's happened and this is what it's going to be like for you.'(Family 4 - daughter) What is suggested here is that such information would help with the fear and worry about the unknown future, and reassurance about any potential worsening of the situation.

\section{Discussion}

This study aimed to explore the difficulties faced, the emotional coping, and the support needs of children, by triangulating data from interviews with children, their parents and support workers. Indeed, in comparing the data from the three groups of participants, our analysis revealed common experiences, observations and suggestions. Three important, and interrelated issues arise from the results of our analysis: the child's experience of complex loss that is difficult to acknowledge, 
emotional distancing between parent and child, and the child's need for credible (i.e peer-based) validation.

Complex loss - i.e. a loss of parental role and personality, but with the person still there. So the person is "there but not there". The loss is denied in various ways the parents may shield the children from it, and the children may be reluctant to acknowledge it. There tends to be more focus on anger in relation to loss rather than sadness (see below for a discussion of this in terms of attachment theory).

Emotional distancing - this partly stems from the complexity of the loss. Parents may feel like they shield their children from the worst aspects of the trauma, perhaps because the parent may be struggling to cope themselves, or be unsure of how to talk to their children about difficult emotions. The children in turn may not want to burden the parent, and keep the loss hidden from their friends and others who they feel would not really understand. They also frequently engage in avoidant or distracting coping strategies. What seems to be created is a gap where children lack some validation for how they feel.

Need for credible validation - the children want to share their experiences with someone who genuinely knows what it is like. But they are stymied by their own emotional distancing strategies (see above). Support workers are frustrated because they feel they are not qualified to support the children, so the workers are aware of their inability to provide credible validation. Parents have to deal with changing roles, and are aware of their own parental absences, leading to reductions in attention to their children.

How does our research relate to previous findings? Like previous research [15] we found there were negative psychological effects of living with a parent with ABI. All children reported some distress, but with no control group it is hard for us to 
disentangle "normal" family stress and stress due to living with a parent with ABI. But there was at least one stress that is most likely to be specific to this group (or any group in which a parent becomes seriously ill): namely, the loss of care and attention from a parent who is still present. This loss is different in kind from that due to death of a parent or divorce. It may share features with both those kinds of losses, but its unique feature is the parent who has changed or who is "there but not there" in some respects.

However, in line with previous research $[1-2,8]$, we found some denial of loss in this group of children. Children in our sample were twice as likely to report anger rather than sadness in relation to their affected parent. And some children explicitly denied any loss when asked, despite mentioning losses elsewhere in their interview. This is interesting because generally research with children from normal populations finds the opposite - that sadness is more easily reported than anger [17]. This could be a quirk of our sample, but it is also plausible that the ambiguity of the loss suffered by these children makes sadness difficult to acknowledge. From an attachment perspective [18], the loss of a parent leads initially to protest (anger) and then to despair (sadness). We propose that the child of a parent with a brain injury may find themselves stuck between attitudes of protest and despair, and often prefer to stay in the protest position. Anger, according to Bowlby [18], consists of more hope than sadness - the initial "protest" (anger) phase of a temporarily abandoned child is trying to summon the parent back, whereas the secondary "despair" (sadness) phase is a kind of giving up or letting go. The logic of despair is that there is no point in wasting energy by protesting if the parent is definitely not coming back.

It follows from this analysis that to be sad about your parent is to acknowledge that something has definitively been lost, but because the loss for children of parents 
with brain injury is ambiguous, it is harder for them to acknowledge it. Children in this case may find it easier to stay in the anger position of trying to reclaim the lost aspects of a parent, rather than the sad position of acknowledging some permanence of the loss.

With regard to coping, we found the most common emotion regulation strategies reported by the children in our sample were suppression, avoidance, distraction, and talking to family members (usually the parent without the brain injury). These findings have limited overlap with those of Kieffer-Kristenson \& Johansen [2]: the main similarity being the high use of distraction, mainly in the form of leisure activities.

In our sample, suppression and avoidance mainly seemed to be associated with hiding away from family stress - children sometimes literally shutting themselves away in their room, or deliberately making sure they didn't cry. These two strategies are usually found to be ineffective or unhelpful in the medium or long term $[10,19]$. Talking to family members and distraction seemed more helpful in our sample, although distraction according to the literature has a mixed efficacy depending on context [9, 20-21]. Distraction can sometimes be a form of avoidance and in these cases a mindful-acceptance strategy (which relaxes the motive to try to control thoughts or emotions) can be more effective [20].

Mindful-acceptance strategies were not reported in our sample, and arguably the most adaptive strategy employed by the children was talking about their feelings. But in line with previous research [1-2], we found that talking about feelings was only with close family members and not with friends, teachers, or support workers. The reason given was overwhelmingly that other people did not truly understand. Indeed, the only one of our sample who did talk to a friend did so because her friend was 
adopted and she felt a commonality: "me and [friend's name] have always got things going on 'cos obviously... [she] has got the whole adoption thing and I've got the brain injured mum" (Family 3 - daughter). But this participant was the only one in our sample who said she had a friend who could understand her situation.

In terms of support needs, previous research has suggested a "need for professionals to talk to the children of injured parents about their experiences of loss and grief' $[2$, p. 8]. However, the reluctance of children to talk openly to such professionals both in our sample and in previous research $[2,8]$ makes this solution problematic. Overwhelmingly in our sample, children expressed a desire to talk to other children in a similar situation to themselves.

Our findings might not be particular to children of parents with an acquired brain injury, but it is difficult to make meaningful comparisons to the experiences of children with parents with other neurological, psychological or medical conditions. This is in part due to the focus tending to be on observing and measuring behavioural aspects of children's responses, with little attention given to the children's subjective experiences [22]. But there are sure to be some overlaps. For example, in a review of the experiences of children of parents with mental health difficulties [22], where it was found that there was an a similar emotional distancing with children being protected by the parents from some of the realities, and children feeling there was no satisfactory outlet for their own range of emotions as parents sometimes felt unavailable to them. The review similarly highlights the need children express to have the opportunity to talk to others they could identify with, which would normalise and validate their experiences. 


\section{Limitations}

There are limitations to our study that need to be acknowledged. Our findings are drawn from a small sample, with differently aged children, and thus cannot be generalised to a broader population. Nevertheless we were able to identify some commonalities in children (and family's) experiences, and were able to substantiate our findings by means of triangulating the views of children, their parents and support workers. The fact that all three groups of participants raised similar things, suggest that these issues are salient for children and families in similar circumstances. However, we can only make these conclusions with caution, given the limits to generalizability. Further research would benefit from drawing on a larger, and more heterogeneous sample. Furthermore, we did not use any objective measures of emotional difficulties (anxiety or depression), coping or impact on functioning in our study, so our findings are based on the reported experiences of children, and the observations of parents and support workers. However, our interest was not in measuring pre-identified specific difficulties and coping strategies, but rather to understand how the children (and parents and support workers) make sense of and interpret their experiences themselves, in keeping with an exploratory approach.

\section{Conclusion}

The key aim of this pilot qualitative study was to investigate the support needs of children whose parents have an acquired brain injury. By interviewing the children themselves, their parents, and support workers, we identified a need for credible peer validation of the children's experiences and feelings. In addition to the provision of information about brain injury and its consequences, the need to talk to someone like them, who will understand what they are going through and can give advice seems of 
utmost importance. A number of useful possible interventions for how this might be achieved were mentioned: by means of lived experience videos; social media support groups (e.g. Facebook); and online and face-to-face peer mentoring. This could provide a fruitful area for further research.

\section{Declaration of Interest}

The authors report no declarations of interest.

\section{References}

[1] Buttera-Prinzi F, Perlesz A. Through children's eyes: children's experience of living with a parent with an acquired brain injury. Brain Injury 2004;18(1):83-101.

[2] Kieffer-Kristensen R, Gaarsvig Johansen KL. Hidden loss: A qualitative explorative study of children living with a parent with acquired brain injury. Brain Injury 2013;27:1562-1569. (doi:10.3109/02699052.2013.841995)

[3] Kieffer-Kristensen R., Teasdale TW, Bilenberg N. Post-traumatic stress symptoms and psychological functioning in children of parents with acquired brain injury. Brain Injury 2011;25:752-760.

[4] Pessar LF, Coad ML, Linn RT, Willer BS. The effects of parental traumatic brain injury on the behaviour of parents and children. Brain Injury 1993;7(3):231-240. 
[5] Uysal S, Hibbard MR, Robillard D, Pappadopulos E, Jaffe M. The effect of parental traumatic brain injury on parenting and child behavior. The Journal of Head Trauma Rehabilitation 1998;13(6):57-71.

[6] LeClere FB, Kowalewski BM. Disability in the family: the effects on children's well-being. Journal of Marriage and the Family 1994; May 1:457-68.

[7] Lazarus RS, Folkman S. Stress, appraisal, and coping. New York: Springer; 1984.

[8] Charles N, Butera-Prinzi F, Perlesz A. Families living with acquired brain injury: A multiple family group experience. NeuroRehabilitation 2007;22(1):61-76.

[9] Mendolia M, Kleck RE. Effects of talking about a stressful event on arousal: does what we talk about make a difference? Journal of Personality and Social Psychology 1993;64(2):283-292.

[10] Aldao A, Nolen-Hoeksema S, Schweizer S. Emotion regulation strategies across psychopathology: A meta-analytic review. Clinical Psychology Review 2010; 30(2):217-237.

[11] Nolen-Hoeksema S, Wisco BE, Lyubomirsky S. Rethinking rumination. Perspectives on Psychological Science 2008;3(5):400-424.

[12] Kozloff R. Networks of social support and the outcome from severe head injury. The Journal of Head Trauma Rehabilitation 1987;2:14-23. 
[13] Evans-Roberts C, Weatherhead S, Vaughan F. Working with families following brain injury. Revista Chilena de Neuropsicología. 2014;9(1):21-30.

[14] Treharne GJ, Riggs DW. Ensuring quality in qualitative research. In: Rohleder P, Lyons AC, editors. Qualitative research in clinical and health psychology. Basingstoke : Palgrave Macmillan; 2015. p 57-73.

[15] Docherty S, Sandelowski M. Focus on qualitative methods: Interviewing children. Research in Nursing \& Health 1999;22(2):177-185.

[16] Braun V, Clarke V, Terry G. Thematic analysis. In: Rohleder P, Lyons AC, editors. Qualitative research in clinical and health psychology. Basingstoke : Palgrave Macmillan; 2015. p 95-113.

[17] Lambie J, Lindberg A. The role of maternal emotional validation and invalidation on children's emotional awareness. Merrill-Palmer Quarterly. In press.

[18] Bowlby J. The making and breaking of affectional bonds. London: Routledge; 1979.

[19] Gross JJ. Emotion regulation: affective, cognitive, and social consequences. Psychophysiology 2002;39(3):281-291. 
[20] Najmi S, Riemann BC, Wegner DM. Managing unwanted intrusive thoughts in obsessive-compulsive disorder: Relative effectiveness of suppression, focused distraction, and acceptance. Behaviour Research and Therapy 2009;47(6):494-503.

[21] Nolen-Hoeksema S, Morrow J. Effects of rumination and distraction on naturally occurring depressed mood. Cognition \& Emotion 1993;7(6):561-70.

[22] Gladstone BM., Boydell K. Children's expriences of parental mental illness: A literature review. Early Intervention in Psychiatry 2011; 5: 271-289. 